\title{
DERECHOS DE AUTOR DE LAS OBRAS REPRODUCIDAS Y PUBLICADAS EN LÍNEA POR LOS MOTORES DE BÚSQUEDA
}

\author{
ERNESTO RENGIFO GARCÍA*
}

\section{i. Presentación}

Los motores de búsqueda reproducen obras en el principal mercado que tienen hoy los autores, esto es, internet. ¿Cómo se trata este fenómeno desde la óptica o bajo la perspectiva del derecho de autor? ¿Constituye ello una infracción al derecho de autor, o bien esos usos pueden ser entendidos como usos justos o leales? La discusión sobre estos interrogantes constituye el tema central de este escrito.

Los motores de búsqueda son sistemas tecnológicos que permiten al usuario de internet explorar y acceder a información. Faye Bohan los define como "herra-

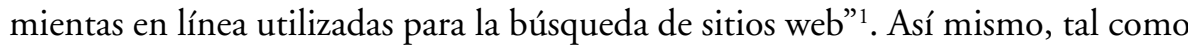
lo ha manifestado James Grimmelman, "[m]ediante la agregación de información sobre las ofertas de los proveedores y presentarla en una forma útil, un motor de búsqueda puede conectar a usuarios con los proveedores de contenidos adecuados, en beneficio de ambos"2.

Ahora bien, como se apreciará en el análisis de la casuística, el concepto ha venido ampliándose, dando cabida a sistemas diseñados para la localización de información y no solo para la mera ubicación de páginas web. Las redes sociales constituyen motores de búsqueda en donde son los usuarios quienes suben con-

\footnotetext{
* Director del Departamento de Propiedad Intelectual y profesor de Derecho de Contratos de la Universidad Externado de Colombia. Abogado asesor y litigante. Este artículo también se publicó en Actes des journées du 20, 21 et 22 octobre 2015, L'effectivité du droit face à la puissance des géants de l'Internet, Bibliothèque de l'IRJS-André Tunc, Institut de recherche juridique de la Sorbonne, t. 74, vol. 2, pp. 281-307. Contacto: [erengifo@ rengifoabogados.com]. Fecha de recepción: 11 de septiembre de 2016. Fecha de aceptación: 30 de noviembre de 2016. Para citar el artículo: Rengifo García, E. Derechos de autor de las obras reproducidas y publicadas en línea por los motores de búsqueda. Revista La Propiedad Inmaterial n. ${ }^{\circ}$ 22, Universidad Externado de Colombia, julio-diciembre 2016 , pp. 33-56. DOI: http://dx.doi.org/10.18601/16571959.n22.03.

1 Bohan Faye, "Liability of Internet Search Engines", Hibernian Law Journal 6, n." 1 (2006), 181-228.

2 James Grimmelman, "The Structure of Search Engine Law”, The University of Iowa Law Review (The University of Iowa) 93 (2007), 1-63.
} 
tenidos, y se accede a ellos mediante los sistemas de ubicación creados por cada una de dichas comunidades sociales; así, por ejemplo, mediante el buscador de Twitter puede accederse a todos los contenidos compartidos por importantes personalidades públicas. Lo mismo sucede con ciertos sitios web dedicados a facilitar la búsqueda académica de artículos en importantes revistas científicas, como es el caso de Heinonline. Es así como, además de herramientas como Google, la evolución tecnológica ha permitido el desarrollo de otros sistemas de investigación que permiten la búsqueda de contenidos, reproduciéndolos o simplemente facilitando el acceso a los mismos a través de mecanismos como el redireccionamiento o el hiperlinking.

Lo cierto es que los motores de búsqueda son intermediarios fundamentales en un mundo cada vez más interconectado. Con ellos se facilita la búsqueda sistemática y ordenada de la información. ¿Qué haría un usuario de la red deseoso de recabar información sobre un tópico particular si no tuviese la ayuda de los buscadores electrónicos? Con razón, en un precedente alemán se señaló que los motores de búsqueda tienen una importancia esencial para estructurar la arquitectura descentralizada de la red, localizando amplios y diseminados contenidos e información $y$, por lo tanto, permitiendo el funcionamiento de una sociedad interconectada ${ }^{3}$.

Los argumentos de defensa cuando los motores de búsqueda publican en línea contenidos protegidos han sido el fair use, el derecho de cita y la licencia implícita, como se verá. Y todo esto, en esencia, por cuanto los representantes de los intereses de los motores de búsqueda sostienen el uso transformativo que hacen de creaciones previas con un alto impacto y beneficio social en una economía de contenidos digitales. ¿El beneficio social que generan los search engines implicará, entonces, que merezcan por parte del derecho de la propiedad intelectual un tratamiento diferente, más flexible y menos riguroso, en perjuicio incluso de los titulares de derechos de autor de los contenidos que inundan la red? No hay que olvidar que internet se ha convertido en el mercado principal de explotación de obras protegidas por el derecho de autor.

\section{BREVE PRECISIÓN TECNOLÓGICA}

Los motores de búsqueda requieren de tres tipos de relación para su funcionamiento. Por un lado, está la relación entre el proveedor de contenido y el motor de búsqueda. Por otro, se encuentra la relación entre el usuario y el motor. Finalmente, se tiene la relación directa entre el usuario y el proveedor de contenidos, la cual es propiciada por el motor de búsqueda. Cada uno de estos tipos de relación

3 " $[E]$ ssential importance for structuring the decentralized architecture of the world wide web, localising widely scattered contents and knowledge, and therefore, ultimately, for the functioning of a networked society": Landgericht Hamburg, case 3080 248/07, citado por Martin Senftleben, "Bridging the differences between copyright's legal traditions - The emerging EC fair use doctrine", 57 J. Copyright Soc'y USA 521 2009-2010, p. 536. 
involucra procesos específicos que son necesarios para que los motores de búsqueda operen adecuadamente.

La primera relación es la que se presenta entre el motor de búsqueda y el proveedor de contenidos. Esta se da a través de dos procesos: la indexación y el caching. Si bien ambos son procesos independientes, sus resultados construyen la base de datos del motor.

La indexación consiste en el proceso mediante el cual el motor de búsqueda "[...] utiliza agentes de software automatizado -robots, arañas o crawlers- para explorar la web y encontrar contenidos. Por lo general, lo hace de la misma manera que un usuario, solicitando las páginas web de los servidores de proveedores

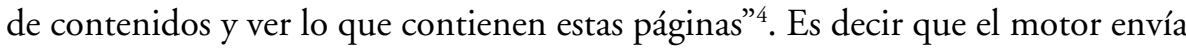
sus spiders a través de la web para identificar contenidos. Hecho esto, elabora un catálogo de toda la información recuperada y lo deja disponible para la próxima vez que un usuario lo solicite.

El caching, por su parte, consiste en la creación de una "copia de la página web [...] tal como lucía cuando los spiders del motor de búsqueda lo visitaron por última vez. Esta copia se mantendrá sin cambios en la memoria caché hasta la próxima vez que la página sea accedida por los spiders" ${ }^{5}$. Se trata entonces de una especie de fotografía cuyo propósito es el de permitir a los usuarios un acceso rápido al contenido en caso de que el sitio web no funcione apropiadamente. Dicha fotografía es conservada en la base de datos del motor hasta que la página sea nuevamente visitada por los crawlers.

La indexación y el caching nutren entonces a los motores de búsqueda. Gracias a estos es posible dar una respuesta inmediata a las consultas presentadas por los usuarios. Sin embargo, son precisamente estas consultas o queries lo que justifica la existencia de los motores. De ahí que la relación entre el usuario y el motor de búsqueda tenga sus propios procesos.

Como se señaló, las consultas son la forma como los usuarios se comunican con el motor de búsqueda. Consisten en "una petición de un usuario para obtener información sobre un tema en particular. La mayoría de los motores de búsqueda usan consultas conformadas por algunas palabras clave o frases cortas. Algunos motores de búsqueda no textuales permiten a los usuarios hacer consultas en formas más exóticas como melodías tarareadas o imágenes" ${ }^{6}$. Así las cosas, los queries son recibidos por el motor y a partir de ese momento revisan toda la información indexada para mostrarle al usuario los temas relacionados con su consulta.

Una vez termina la búsqueda dentro de los temas indexados, el motor arroja los resultados esperados por el usuario. Estos son presentados a través de hipervínculos que dirigen al usuario directamente al sitio web del proveedor de contenidos. "Esta es la etapa en donde los motores de búsqueda realmente se diferencian en-

\footnotetext{
4 Grimmelmann, ob. cit., p. 7-8.

5 FAYE, ob. cit., p. 95.

6 Grimmelmann, ob. cit., p. 8.
} 
tre sí: mediante el uso de variados algoritmos para resumir y organizar los vastos mares de contenido disponible"7. De ahí que se prefieran motores de búsqueda especializados para poder depurar los resultados y encontrar con mayor facilidad los resultados queridos.

Por último, se tiene la relación entre el usuario y el proveedor de contenidos. Gracias a la intermediación del motor de búsqueda es posible que el usuario encuentre los contenidos deseados aun desconociendo inicialmente al proveedor. Sin embargo, estos contenidos no siempre pertenecen al proveedor que los pone a disposición de los usuarios. En esos casos se requiere, además, de contratos de licencia o cesión de derechos de autor para el uso legítimo de los contenidos. En otros casos es posible ampararse en la doctrina del fair use para usarlos.

\section{Derecho norteamericano}

En Estados Unidos, la labor que desempeñan los motores de búsqueda ha encontrado su justificación en la doctrina del fair use. Mejor, el medio de defensa utilizado por los dueños de motores de búsqueda cuando han sido demandados por utilizar contenidos sin previa autorización ha sido el fair use, y en la mayoría de los casos esta defensa ha sido acogida.

Cuando el fair use se esgrime como medio de defensa en un litigio, el juez debe analizar los siguientes cuatro factores: 1) el propósito del uso de la obra originaria dentro de la obra derivada, teniendo en cuenta si se trata de una sátira o una parodia y si el uso es comercial o no comercial; 2) la naturaleza de la obra, mirando, por ejemplo, si es una obra de ficción o si es una obra académica; 3) la cantidad y relevancia de las excertas que han sido tomadas por el trabajo derivado de la obra original; $y, 4)$ el criterio de si la obra transformada le quita mercado o valor a la obra original ${ }^{8}$.

Además de los anteriores factores establecidos en la Sección 107 de la Copyright Act de 1996, la jurisprudencia norteamericana elaboró el criterio del uso transfor-

7 Ibíd., p. 10.

8 El Copyright Act norteamericano señala respecto del uso justo: “ $\$ 107$. Limitations on exclusive rights: Fair use

"Notwithstanding the provisions of sections 106 and $106 \mathrm{~A}$, the fair use of a copyrighted work, including such use by reproduction in copies or phonorecords or by any other means specified by that section, for purposes such as criticism, comment, news reporting, teaching (including multiple copies for classroom use), scholarship, or research, is not an infringement of copyright. In determining whether the use made of a work in any particular case is a fair use the factors to be considered shall include-

"(1) the purpose and character of the use, including whether such use is of a commercial nature or is for nonprofit educational purposes;

"(2) the nature of the copyrighted work;

"(3) the amount and substantiality of the portion used in relation to the copyrighted work as a whole; and

"(4) the effect of the use upon the potential market for or value of the copyrighted work.

"The fact that a work is unpublished shall not itself bar a finding of fair use if such finding is made upon consideration of all the above factors". 
mativo, que al parecer ha venido adquiriendo mayor importancia que los otros cuatro mencionados. Ciertos académicos incluyen dicho factor en el análisis del primer criterio referido, pero varios casos decididos revelan que más que integrar los factores existentes, el uso transformativo se ha convertido en un metafactor, es decir, en el criterio prevaleciente?

\section{Kelly v. Arriba Soft Corp.}

En el tema de los search engines el primer caso a mencionar es Kelly v. Arriba Soft Corp..$^{10}$, en donde se señaló que el uso en los motores de búsqueda de los thumbnails ${ }^{11}$ o imágenes reducidas en respuesta a la información requerida era justo en la medida en que las pequeñas versiones de la imagen original no suplantaban la necesidad de esta, que los thumbnails eran transformativos y que el uso era justo por el public benefit que los motores proporcionan a la sociedad.

El Noveno Circuito sostuvo que el motor de búsqueda en internet llamado Arriba, el cual exhibe sus resultados en la forma de pequeñas imágenes (thumbnails) y no en forma de texto, no violaba el copyright del fotógrafo Leslie Kelly, cuyas imágenes hacían parte de aquellas que aparecían como thumbnails. Kelly argumentó que puesto que Arriba reproducía exactamente sus imágenes y nada agregaba, el uso que hacía no era transformativo; empero la Corte sostuvo lo contrario: "Arriba's use of Kelly's images for its thumbnails was transformative". Para esta autoridad el uso que Arriba hacía de las imágenes servía a una función diferente al uso de Kelly, es decir, se trataba del mejoramiento del acceso a la información en internet versus la expresión artística. Afirmó así que sería improbable que alguien hiciese uso de las imágenes reducidas con propósitos estéticos ya que al ampliarse las imágenes perdían claridad, y que Arriba no reemplaza la obra original, sino más bien había creado una finalidad diferente para las imágenes y por ello el uso que había hecho era transformativo ${ }^{12}$.

9 Ver Matthew D. Bunker y Clay Calvert, "The Jurisprudence of transformation: Intellectual Incoherence and Doctrinal Murkiness Twenty Years after Campbell v. AcuffRose Music", Duke Law \& Technology Review, vol. 12, 2014, 92-128. "One suggestion is returning the doctrine to a more modest role in fair-use cases. Transformative use, which almost certainly should include uses that add new meaning or message even without referring to the borrowed work, could operate once again as the Campbell Court seemed to intent it, as an ameliorating device in cases of commercial borrowing, rather than as a meta-factor that seems to trump all other considerations. This sort of 'transformative minimalism' seems more in keeping with the spirit of the statute rather than the bloated doctrine now dominating the scene post-Campbell' (p. 127).

10 Kelly v. Arriba Soft Corp., 336 F.3d 811, 817-22 (9 $9^{\text {th }}$ Cir. 2002).

11 "Un Thumbnail es una versión de menor resolución (y por lo tanto más pequeña) de una imagen de tamaño completo. Las miniaturas permiten a los usuarios procesar y ubicar rápidamente información visual [...] Como las miniaturas son más pequeñas, pueden exhibirse más al mismo tiempo en una sola página o pantalla. Los usuarios pueden examinar rápidamente el conjunto completo de miniaturas para ubicar la imagen particular en tamaño completo que están buscando” (CERLALC, 2010).

12 "Arriba's use of the images serves a different function than Kelly's use-improving access to information on the internet versus artistic expression. Furthermore, it would be unlikely that 
Por supuesto que una decisión de este talante podría originar escozor a un jurista del civil law: ¡cómo así que hay uso justo no obstante una reproducción exacta de la creación original?, pero el beneficio social, en razón del nuevo uso que proporciona el motor de búsqueda, arrastra ese nuevo uso, derivado de una obra protegida (las fotografías), a la categoría del fair use. Es así de sencillo.

\section{Caso Perfect 10}

La lógica expuesta es recogida en el caso Perfect $10 \mathrm{Inc}$. v. Amazon y Google Inc. $(2007)^{13}$. Este caso, junto con el de Arriba, son inicialmente, en la jurisprudencia norteamericana, los más representativos en favor de los motores de búsqueda porque con ellos se justifica su funcionamiento e indiscutible utilidad en la red: permiten el acceso organizado de la información.

Perfect 10 es una revista muy similar a Playboy. Google, como parte de su servicio de búsqueda de imágenes, hace una reducción de las imágenes, copias en miniatura o thumbnails para permitir que vayan direccionadas a un sitio donde el usuario puede verlas completas. Perfect 10 demandó a Amazon y a Google por estar reproduciendo ilícitamente las imágenes sobre las cuales tiene copyright $t^{14}$. El juez consideró que no había violación del copyright porque Google ofrecía un nuevo instrumento que permitía el acceso a nuevos contenidos. En efecto, el propósito del uso de las obras había cambiado para convertirlas en mecanismos de búsqueda e información a través de motores de búsqueda: "a search engine transforms the image into a pointer directing a user to source of information". El uso de imágenes en forma reducida constituye "a new work, namely, an electronic reference tool".

En concreto, la doctrina del fair use pretende garantizar el equilibrio de intereses y derechos. Por ello, en el caso Perfect 10, al concluirse que transformando la obra artística en un instrumento de referencia electrónica se proporciona un beneficio a la sociedad, se está acogiendo el criterio del beneficio social, el cual, a la luz del derecho norteamericano, constituye uno de los elementos basilares que subyacen en la filosofía del fair use.

La Corte de Apelaciones del Noveno Circuito consideró que emplear las fotografías en miniatura (thumbnail images) de Perfect 10 como links o enlaces a otros sitios web era un uso justo permitido por la ley. La Corte sostuvo:

... we now weigh these factors together "in light of the purposes of copyright". [...] In this case, Google has put Perfect 10 thumbnail images (along with millions of other thumbnail

anyone would use Arriba's thumbnails for illustrative or aesthetic purposes because enlarging them sacrifices their clarity. Because Arriba's use is not superseding Kelly's use but, rather, has created a different purpose for the images, Arriba's use is transformative".

13 Perfect 10, Inc. v. Amazon.com, Inc., 508 F.3d 1146, 1163-68 (9 $9^{\text {th }}$ Cir. 2007).

14 Una explicación del caso se puede hallar en Óscar Ramírez, El equilibrio entre la propiedad intelectual y el acceso a la información y tecnología desde la perspectiva del usuario, tesis de Maestría, Bogotá, Universidad Externado de Colombia, 2015, p. 128-130. 
images) to a use fundamentally different than the use intended by Perfect 10. In doing so, Google has provided a significant benefit to the public. Weighing this significant transformative use against the unproven use of Google's thumbnail for cell phone downloads, and considering the other fair use factors, all in light of the purpose of copyright, we conclude that Google's use of Perfect 10's thumbnails is a fair use.

Para la Corte, incluso, un motor de búsqueda puede ser más transformativo que una parodia en la medida en que proporciona un nuevo uso de una forma original, mientras que la parodia tiene el mismo propósito de entretenimiento de la obra originaria $^{15}$.

Obsérvese entonces que el beneficio social se coloca incluso por encima de los derechos del titular para justificar esa reproducción exacta pero reducida de la creación original, y de esta manera se justifica la labor que cumplen los motores de búsqueda en la red. En otras palabras: el beneficio social prevalece sobre la violación del derecho de autor y por ello encuentra su justificación en la doctrina del fair use. Se lee en el fallo: "The significantly transformative nature of Google's search engine, particularly in light of its public benefit, outweighs Google's superseding and commercial uses of the thumbnails in this case. In reaching this conclusion, we note the importance of analysing fair use flexibility in light of new circumstances" 16 .

El uso de las imágenes reducidas con propósitos de búsquedas es altamente transformativo y por ello no se puede presumir un daño en el mercado. Es decir que el uso de imágenes reducidas y de baja resolución no son mercados sustitutos de las imágenes originarias.

Lo que se observa, entonces, es que en el derecho norteamericano existe una actitud de tolerancia y de protección a los motores de búsqueda ${ }^{17}$. Esto es indiscutible. Hacer una copia exacta de una creación precedente es transformativo en la medida en que la copia tiene una función diferente a la obra original (!) ("so long as the copy serves a different function than the original work") y las imágenes reducidas no son mercados sustitutos de las imágenes originales.

Los motores de búsqueda juegan un rol trascendental en la diseminación y en el acceso a la información y al conocimiento. Con la decisión de Perfect 10 se fortalece la función de los motores de búsqueda de promover el conocimiento público y con ella se continúa la tendencia de interpretar de una manera liberal y abierta la doctrina del fair use ${ }^{18}$.

15 "Indeed, a search engine may be more transformative than a parody because a search engine provides an entirely new use for the original work, while a parody typically has the same entertainment purpose as the original work": Perfect 10, Inc, v. Amazon.com, 487 F.3d 701,721 ( $9^{\text {th }}$ Cir. 2007)

16 Perfect 10, Inc, v. Amazon.com, 487 F.3d 701, 723 (9 ${ }^{\text {th }}$ Cir. 2007).

17 Ver Амч Виом, Search Engines and paragraph 512 (D) of the D.M.C.A, 1 Case W. Res. J.L. Tech. \& Internet 36, 2009-2010.

18 Ver Meng Ding, "Perfect 10 v. Amazon.com: A Step Toward Copyright's Tort Law Roots”, Berkeley Technology Law Journal, vol. 23, n.o 1, February 2014. 
Associated Press v. Meltwater Holding (2013). Meltwater es una multinacional con sede en Noruega, dedicada al negocio del software as a service que ofrece el servicio de noticias en Estados Unidos con el uso de keywords; para ello utiliza "automated computer programs or algorithms to copy or 'scrape' an article from an online news source, index the article, and deliver verbatim excerpts of the article" ${ }^{19}$. Es decir que el software de Meltwater automáticamente realizaba el resumen de los artículos periodísticos de Associated Press (AP). La Corte consideró que el software, al hacer un resumen de los artículos de los medios asociados a AP, infringía el copyright. Aquí se consideró que el uso de los contenidos no era transformativo en la medida en que el material de la AP era efectivamente substituido en el mercado por los resúmenes del demandado. La Corte, en efecto, identificó a Meltwater como competidor de AP y de sus licenciatarios.

Meltwater argumentó que él operaba como un motor de búsqueda y por ello el uso que hacía de la información de la Ap era un uso justo. Sin embargo, la Corte sostuvo lo contrario, en el sentido de que no había transformación de la información para facilitar su acceso, sino que el uso que hacían los usuarios del servicio de Meltwater suplantaba las necesidades del artículo noticioso original, "instead of driving subscribers to third-party websites, Meltwater News acts as a substitute for news sites operated or licensed by AP", y que "the purpose and character of Meltwater's use of AP's articles weigh against a finding of fair use".

La Corte encontró, entre otras cosas, que el cuarto factor del fair use -el criterio de si la obra transformada le quita mercado o valor a la obra original- actuaba en contra de Meltwater porque AP concedía licencias y obtenía pagos de negocios ubicados dentro del mismo mercado en donde actuaba Meltwater. El modelo de negocios del demandado competía directamente con el del demandante y aquel no pagaba regalías o derechos por licencia-licensingfees-. Para la Corte fue irrelevante que Meltwater utilizara la tecnología de los motores de búsqueda ya que el demandado debió probar que el uso del contenido era justo, cosa que no aconteción ${ }^{20}$.

Conviene destacar de este caso que Meltwater esgrimió que Ap había concedido una licencia implícita para usar sus contenidos. Argumentó el demandado que los licenciatarios de AP no emplearon robots.txt -un protocolo que permite a los sitios web comunicarles a los rastreadores de Meltwater no usar el contenido-, que AP había adquirido y licenciado en forma no exclusiva a Meltwater para redistribuir

19 Associated Press v. Meltwater U.S. Holdings, Inc., 931 F. Supp. 2d 537, 540 (s.D.N.Y. 2013).

20 "The private nature of Meltwater's service and the presence of licensing agreements between AP and similar parties played a significant role in Judge Cote's reasoning. At later steps in the analysis, she found the use of lades and excerpts did not constitute a 'transformative use', and the weighing of the public interest favoured protection of AP's rights": Rosalina Jane Schonwald, "Associated Press v. Meltwater US Holdings, Inc.; Fair Use, a Changing News Industry, and the Influence of Judicial Discretion and Custom”, 29 Berkeley Tech. L.J. 799 2014, p. 818-819. 
sus artículos protegidos. Frente a este argumento la Corte señaló que AP no había otorgado una licencia implícita al demandado por cuanto los requisitos para que esta figura opere en el derecho norteamericano no estaban satisfechos. Pero la Corte, en esencia, no aceptó esta defensa porque ello implicaría un cambio en la carga de la prueba en el sentido de que, alegada la licencia implícita, el titular debería demostrar que no la otorgó, cuando la lógica es que si el titular alega la infracción de su derecho el onus probandi está en cabeza del presunto infractor ${ }^{21}$, quien deberá demostrar que ha usado el contenido de manera legal.

Si bien este argumento no prosperó, es conveniente mencionarlo, así sea de manera sumaria, porque -como se verá infra- sería luego utilizado en un caso alemán para justificar la actividad de los motores de búsqueda ante la inflexibilidad del régimen de limitaciones que opera en el derecho europeo.

\section{Caso Google Books}

En el caso The Authors Guild, Inc. and Betty Miles, Joseph Goulden, and Jim Bouton v. Google Inc. ${ }^{22}$, los titulares demandaron a Google por cuanto para ellos el Library Project y el Google Books Project infringían sus derechos de autor. A través de estos proyectos, Google hace y retiene copia de los libros, permite que las bibliotecas descarguen y retengan una copia digital, y facilita que el público busque los textos o los libros digitalmente copiados o reproducidos y pueda ver representaciones de snippets del texto. Los libros, pues, son escaneados por Google sin previa autorización y son puestos a disposición de los usuarios de internet en snippet view en el sitio web de Google. Lo que perseguía la acción era que Google removiese esa vista recortada o tijereteada de los libros.

La primera instancia analizó los cuatro factores de la Sección 107 y consideró, en esencia, que los usos a las obras que realizaba Google eran transformativos, que la exhibición del material era limitada y que el Google Books Program no constituía un mercado substituto de las obras originales. El juez del Distrito de New York exoneró a Google por cuanto, aun en presencia de reproducción de la obra, su finalidad es la de facilitar la búsqueda de libros por internet. Así, entonces, este motor de búsqueda ofrece información de los libros a través de extractos, o snippets, los cuales no constituyen un instrumento que suplante al libro, sino un medio que facilita su acceso; por lo tanto produce un alto beneficio social y no hay violación del copyright.

Para el momento en que se profirió esta decisión surgieron ciertas reflexiones: ¿qué acontece con el cambio de formato?, ¿qué sucede con la digitalización de las

21 "[W]hat Meltwater is suggesting would shift the burden to the copyright holder to prevent unauthorized use instead of placing the burden on the infringing party to show it had properly taken and used content". Para un análisis crítico de esta sentencia véase también el comentario de Dylan Quinn, 15 Minn. J.L. Sci. \& Tech. 11892014.

22 Authors Guild, Inc. v. Google Inc., 954 F. Supp 2d 282, 291 (s.D.N.y. 2013). 
obras expresadas en formatos tradicionales? A la luz del derecho continental se podría decir que un cambio de formato es una forma de reproducción y por lo tanto no se puede hacer sin autorización del titular; en cambio, a la luz del beneficio social y del fair use la digitalización es permitida. La autoridad norteamericana se refirió al beneficio social y a las ventajas que originaba un proyecto como Google Books; a la dinámica en las bibliotecas; al data mining o test mining (minería de datos), esto es, al proceso de extraer elementos interesantes o categorías conceptuales que un escritor utiliza y crear un perfil para saber en qué tendencia literaria se ubica ese autor; al acceso y conservación de la literatura en el tiempo; a la publicación a través de fragmentos, y todo esto para aplicar en favor de Google el fair use. Dice el fallo que Google Books no suplanta al libro porque es un instrumento para utilizar libros y, además, porque esta nueva herramienta indica en qué lugar y en qué librería se podría encontrar el libro en físico.

Sin embargo, el tema de la digitalización del libro sin autorización del autor y de los editores seguía siendo materia de debate y más a la luz del derecho norteamericano, en donde un famoso precedente de la Suprema Corte en el caso Tasini había señalado que cada vez que un editor quiera colocar en una base de datos digital obras de los autores, requiere autorización previa ${ }^{23}$. Entonces la pregunta que surgía era si esta decisión de primera instancia de un juez de distrito estaba en contradicción con este precedente de la Suprema Corte.

Al parecer, el concepto del transformative use comprende no solo la modificación o alteración sustancial del contenido, sino además el cambio de formato, cuando este satisfaga las finalidades del derecho de autor y los supuestos de aplicación de la doctrina del fair use; es decir, el cambio de formato que facilite el acceso a los contenidos caería en la doctrina del fair use y, por lo tanto, no habría violación del copyright.

Google convierte el libro en información, por lo que hace un uso transformativo de la obra original, y, al haberla transformado para beneficio social, cae en uso justo: "Google Books is also transformative in the sense that it has transformed book text into data for purposes of substantive research, including data mining and text mining in new areas".

En conclusión, el cambio del texto a un formato digital implica un uso transformativo, y el proyecto de digitalización de Google implica un beneficio masivo para la sociedad. "Google Books digitizes books and transforms expressive text into a comprehensive word index that helps readers, scholars, researchers, and others find books. [...] Google books does not supersede or supplant books because it is not a tool to be used to read books", se lee en la sentencia ${ }^{24}$.

23 New York Times Co. v. Tasini, 533 U.S. 483, 121 S. Ct. 2381, 150 L. Ed. 2d 500 (2001). En la sentencia se lee: "We further conclude that the Print Publishers infringed the Authors' copyrights by authorizing the Electronic Publishers to place the Articles in the Databases and by aiding the Electronic Publishers in that endeavor" (p. 506).

24 Un profesor norteamericano comentaba así esta primera decisión: "Let me emphasize, this is a case about more than twenty million books. This is not about scanning one book 
En la segunda instancia, la Corte de Apelación para el Segundo Circuito, el 16 de octubre de $2015^{[25]}$, confirmó la decisión de la primera instancia. De este fallo y para los efectos de este escrito vale la pena mencionar sus principales consideraciones, comenzando por esta: no importa cuánto se haya copiado, sino cuánto termina puesto a disposición o accesible al público. Es decir que, como lo que termina a disposición del público son solo snippets, entonces no habría vulneración. Obsérvese la lógica del sistema: la reproducción previa que sirve de base al snippets no cuenta, lo relevante es que como estos no ofrecen la reproducción total de la obra, entonces no hay violación del derecho (!).

La Corte de Apelaciones concluyó que la copia que hace Google es transformativa en la medida en que no es un meaningful substitute de las obras protegidas de los demandantes y satisface el test del fair use de la Sección 107 del Copyright Act.

La Corte de Apelaciones, al analizar la doctrina del fair use, sostiene que esta permite, bajo ciertas circunstancias, que una obra previa pueda ser copiada en la medida que el propósito del copyright es el de promocionar el progreso de la ciencia y de las artes útiles. Respecto del primer factor señala que los usos transformativos tienden a favorecer el fair use porque "a transformative use is one that communicates something new and different from the original or expands its utility, thus serving copyright's overall objective of contributing to public knowledge". La idea del beneficio social o al público impregna la doctrina del uso justo. En el caso, el snippet view tiene un alto valor transformativo porque identifica el interés del libro para el usuario o buscador.

Para los demandantes, el interés comercial de Google actuaría en su contra, es decir que por ello el uso sería injusto. Sin embargo, puesta frente a este poderoso escollo conceptual la Corte lo elude así:

Congress could not have intended a rule that commercial uses are presumptively unfair. Instead, the more transformative the new work, the less will be the significance of others factors, like commercialism, that may weigh against a finding of fair use. [...] we see no reason in this case why Google's overall profit motivation should prevail as a reason for denying fair use over its highly convincing transformative purpose, together with the absence of significant substitute competition, as reason for granting fair use. Many of the most universally accepted forms of fair use, [...], are all normally done commercially for profit.

and arguing with one author. This is about scanning millions of books and permitting public search, which the court ruled was transformative and within fair use, in large part because of these 'new uses' to which it could be put, and the new uses are searchability and, the ability to use digitized books and reach readers who are visually impaired and need the benefit of seeing it on the screen. Then in the end, the court's resolution that 'Google's actions [provide] the libraries with the ability to engage in activities that advance the arts and sciences,' is constitucionally grounded. The court was harkening back to that conceptual language in the U.S. Constitution": Kenneth D. Crews, "The Role of the Judge in Common Law Countries When Deciding Fair Use Cases", Revista Iberoamericana de Derecho de Autor, Bogotá, Cerlalc, año viII, n. ${ }^{\circ}$ 15, enero-junio 2015, p. 61.

25 Authors Guild, Inc. v. Google, Inc., n.o 13-4829-cv (2d Cir. Oct. 16, 2015). 
Así pues, el hecho de que Google ofrezca digitalmente una obra significativamente transformada con propósitos económicos no impide la aplicación del uso justo.

Respecto del segundo factor, esto es, del relacionado con la naturaleza de la creación protegida, es decir, si es una obra de ficción o una obra académica, en donde en principio sería más fácil deducir el uso justo when the copying is offactual works than when copying is from works of fiction, la Corte señaló que si bien en el caso se estaban discutiendo tres libros "factuales" de los demandantes, este criterio no es fundamental para determinar el fair use en favor de Google. Mejor dicho, el segundo factor favorece a Google no porque las obras de los demandantes sean de carácter fáctico, sino porque el uso transformativo secundario proporciona información valiosa acerca del libro original, más que ser una réplica de la expresión protegida; es decir que la versión digital de Google es un substituto significativo del origina $^{26}$. Aquí es importante destacar también que para la Corte el carácter fáctico del libro original no justifica la copia o reproducción de la expresión protegida.

Respecto del tercer factor, esto es, el relacionado con la cantidad de material tomado de la obra original, criterio cuantitativo que sirve para dilucidar si el uso es justo o no, la Corte de Apelaciones afirmó que es más fácil hallar la limitación del uso justo cuando se toman pequeñas partes del trabajo protegido, pero como en el caso lo que hace Google es copiar digitalmente unos trabajos previos, afirmó que la copia íntegra o total de un trabajo es en algunos casos necesaria para hacer que el uso justo sea viable o procedente ("copying the entirety of a work is sometimes necessary to make a fair use of the work"). Es decir que, como en la función de búsqueda (search function) Google tiene que reproducir integralmente el trabajo, la Corte rechazó el argumento según el cual la copia total de un trabajo no puede ser considerada un uso justo. "While Google makes an unauthorized digital copy of the entire book, it does not reveal that digital copy to the public. The copy is made the search functions to reveal limited important information about the books. With respect to the search function, Google satisfies the third factor test".

Ahora, respecto de la visión recortada, tijereteada o snippet view, la Corte indicó que esta no ofrece problemas al mercado en la medida que no constituye un producto sustituto significativamente competitivo para el trabajo original y que la información que proporciona no satisface las necesidades del usuario o busca$\operatorname{dor}^{27}$. Y agregó que la información que revela no es sustancial, en el sentido de que comunica poco respecto del sentido de la obra original: "The fact that Plaintiffs"

26 "[T]he second factor favours fair use not because Plaintiffs' works are factual, but because the secondary use transformatively provides valuable information about the original, rather than replicating protected expression in a manner that provides a meaningful substitute for the original': ibíd.

27 "We nonetheless conclude that, at least as presently structured by Google, the snippet view does not reveal matter that offers the marketplace a significantly competing substitute for the copyrighted work. [...] In addition, Google does not provide snippet view for types of books, such as dictionaries and cookbooks, for which viewing a small segment is likely to satisfy the searcher's need": ibíd. 
searchers managed to reveal nearly $16 \%$ of the text Plaintiff' books overstates the degree to which snippet view can provide a meaningful substitute".

En cuanto al cuarto factor, esto es, el efecto que la copia produciría en el mercado o en el valor de la obra protegida (al parecer el factor más importante para decidir este caso), la Corte consideró que la función snippet no daba un acceso efectivo a un substituto competitivo ${ }^{28}$, puesto que solo permitía vistas discontinuas o pequeños fragmentos que no iban más allá del 16\% del total del libro. "This does not threaten the rights holders with any significant harm to the value of their copyrights or diminish their harvest of copyright revenue".

Ahora bien, no obstante que la Corte reconoce que esas vistas parciales pueden afectar económicamente al autor, acepta la limitación. Este es quizá uno de los puntos más sensibles y destacables del fallo. Es decir que, si bien subsiste la probabilidad del daño, en el sentido de que los buscadores pueden preferir esa versión recortada y no la integral del libro, la Corte acepta el fair use porque dicha versión recortada no es suficiente para descartarlo, o mejor, porque esa vista no integral no constituye una copia sustituta y competitiva de la obra original que pueda afectar el mercado o su valor como obra protegida ${ }^{29}$. Además porque el posible efecto en el mercado debe ser relevante y significativo.

La Corte, pues, concluyó que hacer una copia digital integral de las obras de los demandantes con el propósito de proporcionar al público su búsqueda y realizar un diseño que permite una visión limitada o recortada de la obra constituye un fair use que no infringe los derechos de autor de las obras.

Obsérvese que las copias son usadas para permitir que el público obtenga información acerca de los libros. Entonces, ante esto queda el interrogante sobre qué acontece con la obra derivada. Esto fue analizado por la Corte y constituye en últimas la justificación de la digitalización de las obras sin autorización del titular de la obra original, e incluso, yendo más lejos, lo que justificaría el actuar de los motores de búsqueda en internet (es decir, el lugar que en la actualidad se ha convertido en el principal mercado de explotación de obras), una postura en desmedro de autores y titulares de derechos: "The copyright resulting from the Plaintiffs's authorship of their works does not include an exclusive right to furnish the kind of information about the works that Google's programs provide to the public. For substantially the same reasons, the copyright that protects Plaintiffs' works does not include an exclusive derivative right to supply such information through query of a digitized copy".

28 "[T] he snippet function does not give searchers access to effectively competing substitutes".

29 "We recognize that the snippet function can cause some loss of sales. There are surely instances in which a searcher's need for access to a text will be satisfied by the snippet view, resulting in either the loss of a sale to that searcher, or reduction of demand on libraries for that title, which might have resulted in libraries purchasing additional copies. But the possibility, or even the probability or certainty, of some loss does not suffice to make the copy an effectively competing substitute that would tilt the weighty fourth factor in favour of the rights holder in the original. There must be a meaningful or significant effect upon the potential market for or value of the copyrighted work"'. 
En corto: sostiene y dictamina la Corte que la información relacionada con el libro no es monopolio del titular, sino que otro sujeto la puede suministrar. Es decir que la información en formato digital no es monopolio del titular de la obra informada. En fin, que el cambio de formato, esto es, la digitalización de la obra ya no es monopolio del creador de la obra original. La decisión representa un gran alivio para los explotadores de contenidos y un golpe contundente para los titulares de los derechos.

Sin embargo, el punto no es tan claro. En efecto, la Corte de Apelación señaló: "The extension of copyright protection beyond the copying of the work in its original form to cover also the copying of a derivative reflects a clear and logical policy choice. An author's right to control and profit from the dissemination of her work ought not to be evaded by conversion of the work into a different form". Esto es, el titular de la obra tiene derecho a todas las obras que se derivan de la misma: traducciones, arreglos, resúmenes, dramatizaciones, etc.

Una obra no se puede digitalizar sin autorización de su creador. Entonces, ante esa dificultad del derecho a la obra derivada que tiene el titular, la Corte recurre a una sutileza para decir que en el caso no se está frente a una obra derivada, sino frente a información digital de la obra original y sobre la cual no tiene un derecho exclusivo el titular de dicha obra:

If Plaintiffs claims were based on Google's converting their books into a digitized form and making that digitized version accessible to the public, their claim would be strong. But as noted above, Google safeguards from public view the digitized copies it makes and allows access only to the extent of permitting the public to search for the very limited information accessible through the search function and snippet view. The program does not allow access in any substantial way to a book's expressive content. Nothing in the statutory definition of a derivative work, or of the logic that underlines it, suggests that the author of an original work enjoys an exclusive derivative right to supply information about that work of the sort communicated by Google's search functions.

Nótese la sutileza: una cosa es la obra derivada, la cual, por supuesto, hace parte de los derechos exclusivos del autor, y otra es la información relacionada con la obra original, la cual no se halla dentro del alcance de los derechos exclusivos. Y para la Corte, como el programa de Google no permite un "acceso sustancial" a los contenidos protegidos, no hay vulneración de derechos. No existe, pues, un derecho derivado en el suministro de información sobre una obra que provee Google en sus funciones de búsqueda (Googles search functions). Esta afirmación representa un aval o, mejor incluso, una trascendental justificación teórica, jurídica y económica a la actividad que despliegan los motores de búsqueda en la medida en que la reproducción integral previa se halla amparada por la doctrina del fair use.

Para los demandantes la digitalización previa debería ser materia de licenciamiento; empero para la Corte ello no es necesario en la medida en que lo que 
hace Google es permitir que el público obtenga información relacionada con el contenido de los libros, sin permitir una lectura sustancial de los textos (without allowing any substantial reading of its text).

La Corte concluyó, entonces, que la digitalización no autorizada de obras protegidas, la creación de una funcionalidad de búsqueda y la exhibición de snippets de trabajos protegidos son "non-infringing fair uses"; que en el caso sub examine el propósito de la copia es altamente transformativo; que la exhibición pública del texto es limitada; que las revelaciones no implican un significativo mercado sustituto de los aspectos protegidos de las obras originales, y que la naturaleza comercial y la motivación de ganancia de Google no justifican rechazar la limitación del fair use.

La validez de esta decisión a la luz de la experiencia jurídica del derecho continental tendría grandes escollos teóricos y conceptuales para ser aceptada, y quizá podría ser justificada más por razones económicas que por consideraciones de estricto rigor jurídico. Pero en general se observa la extraordinaria flexibilidad interpretativa que tiene cada uno de los criterios que conforman el fair use, flexibilidad aprovechada precisamente para acompasar el derecho de autor con el desarrollo de las tecnologías de la información, la economía y entorno digital. Que el dilema autor/explotador se suele resolver, por consideraciones de mercado, económicas y con una alta dosis de utilitarismo anglosajón, en favor del segundo. Y que " $[t]$ he broad standard of 'fair use' and its discretionary application allows the courts to serve the purpose of U.S. copyright law while also considering the public interest to a sustainable degree" 30 .

Así pues, la flexibilidad que tiene la doctrina del fair use es lo que le ha permitido a la jurisprudencia norteamericana avalar la labor que los motores de búsqueda realizan en una sociedad cada vez más interconectada y en permanente intercambio de grandes cantidades de información digitalizada.

En resumen, y tal como ya se mencionó, no importa cuánto se haya copiado, lo importante es cuánto termina puesto a disposición o accesible al público. Como el snippet no contiene una parte sustancial de la obra protegida, no existe vulneración.

Finalmente ha de observarse que en la primera instancia el abogado de Authors Guild había aducido que la evaluación del caso no debería estar basada en los beneficios que los usuarios obtienen de Google Books, sino en las ventajas competitivas y ganancias económicas obtenidas por Google como consecuencia del uso de las obras sin previa autorización de los autores y sin el debido reconocimiento pecuniario. Sin embargo, esta circunstancia, de la ganancia obtenida por Google, tampoco afectó el reconocimiento del uso justo ${ }^{31}$.

30 Florian Pötzlberger, "Google and the Thumbnail Dilemma- 'Fair use' in German Copyright Law?” 9 ISJLP 139 2013-2014, p. 146.

31 Herrera Sierra, Luisa Fernanda, “Estudio del Caso Google Books y efectos del fair use en el entorno digital”, disponible en: http://propintel.uexternado.edu.co/estudio-delcaso-google-books-y-efectos-del-fair-use-en-el-entorno-digital/ 


\section{Derecho europeo}

En términos generales se puede decir que el derecho continental europeo no cuenta específicamente con una limitación aplicable a los motores de búsqueda y, por lo tanto, no estarían exonerados; sin embargo, los expertos se preguntan si el derecho de cita (right of quotation) sería una solución viable.

En Alemania, en el año 2010, la Corte Federal de Justicia, en un caso en donde un artista reclamaba que Google infringía su derecho de autor al exhibir en versión reducida (thumbnails) su trabajo en la lista de resultados, consideró que cualquier publicación de thumbnails constituía infracción al derecho de autor de acuerdo con el parágrafo 19a de la Ley alemana de derechos de autor. La Corte confirmó el análisis de la Corte de Apelación en el sentido de que no había excepción aplicable que justificara la actividad de Google. Sin embargo, la Corte Federal estimó que la infracción estaba justificada porque el titular aceptó la conducta constitutiva de la vulneración. La Corte Federal, en efecto, concluyó que cargar o alimentar imágenes en internet, sin implementar ninguna restricción técnica que permita bloquear la función de los motores de búsqueda, constituye un consentimiento implícito en el uso de esas obras. Es decir que esta autoridad recurrió a una ficción legal derivada del sistema general del derecho -el consentimiento implícito- para darle una salida a la actividad de los motores de búsqueda.

Para el Bundesgerichtshof la excepción del derecho de cita no aplica como justificación a los servicios que prestan los buscadores de imagen:

[N]either the technical developments concerning the dissemination of information on the Internet not the interest of the parties which the exception seeks to protect justify an extensive interpretation of paragraph 51 of the German Copyright Act that goes beyond the purpose ok making quotations. Neither the freedom of information of other Internet users, nor the freedom of communication or the freedom of trade search engine providers, require such an exclusive interpretation ${ }^{32}$.

Por ello, entonces, la Corte Federal de Alemania acogió la alternativa del consentimiento implícito, y con ello creó "un espacio de respiro" para los servicios de búsqueda de imágenes en la red ${ }^{33}$.

Lo que se percibe de esta decisión es que el sistema rígido de las limitaciones o excepciones no permite exonerar a los motores de búsqueda de imágenes en la red y entonces, para justificar esta actividad, la Corte alemana hubo de hechar mano

32 Ver BGH, Apr. 29, 2010, case I ZR 69/08, at 11-12, citado por Martin Senftleben, "Bridging the differences between copyright's legal traditions - The emerging EC fair use doctrine", 57 J. Copyright Soc'y U.S.A. 521, 2009-2010.

33 "[T]he Court held that Google's use of the pictures was not unlawful because the copyright owner had consented implicitly to use of her material in the image search service by making her works available online without employing technical means to block the automatic indexing and displaying of online content by search engines": Senftleben, ob. cit., p. 537 y 538. 
del consentimiento implícito, es decir, de una figura propia del régimen común de los contratos - pero no del régimen especial del derecho de autor- como lo es la figura de las licencias contractuales implícitas.

Senftleben sostiene que esta decisión es de particular interés porque prueba la actual estructura restrictiva del derecho de las limitaciones en la Unión Europea. Para él, el concepto híbrido de las precisas excepciones legales y la regla de los tres pasos no ofrecen suficiente espacio a las cortes para maniobrar, y por ello hay que recurrir a vías alternas para llegar a resultados satisfactorios. Así, la asunción del consentimiento implícito aparece como un intento para evitar la inflexible infraestructura de las limitaciones al derecho de autor. De todas maneras, critica que esa solución sea consistente y cuestiona la introducción de ese elemento flexible (consentimiento implícito) por la puerta de atrás de las dudosas presunciones de intención de un titular de derechos que pone a disposición su creación en internet ${ }^{34}$.

Es decir que como el uploader no protege el contenido que pone a disposición en la red, está implícitamente autorizando su uso por los motores de búsqueda; esta postura es en cierta medida equivalente a la utilizada por el Tribunal de Justicia Europeo, en su interpretación prejudicial del 13 de febrero de 2014 en el caso Svenson ${ }^{35}$, en el sentido de que como no existe un "público nuevo", la comunicación al público no exige la autorización de los titulares de los derechos de autor ${ }^{36}$.

34 Cfr. ibíd., p. 538: "The German Federal Court of Justice introduced a flexible element through the back door of doubtful assumptions of the intentions of a copyright owner making her works available on the internet".

35 “[L]a presentación en una página de internet de un enlace sobre el que se puede pulsar y que conduce a una obra protegida publicada y libremente accesible en otra página de internet tiene por efecto poner dicha obra a disposición de los usuarios de la primera página mencionada y constituye, por tanto, una comunicación al público. No obstante, dado que no existe un público nuevo, en todo caso tal comunicación al público no exige la autorización de los titulares de los derechos de autor. Por el contrario, en el caso de que el enlace sobre el que se puede pulsar permitiera a los usuarios de la página en la que se encuentra dicho enlace eludir las medidas de restricción adoptadas en la página en la que se encuentra la obra protegida para limitar el acceso a ésta a los abonados y constituyera, de este modo, una intervención sin la cual dichos usuarios no podrían disfrutar de tales obras difundidas, habría que considerar que el conjunto de esos usuarios es un público nuevo que no fue tomado en consideración por los titulares de los derechos de autor cuando autorizaron la comunicación inicial, de modo que tal comunicación al público exigiría la autorización de los titulares. [...] No constituye un acto de comunicación al público [...] la presentación en una página de internet de enlaces sobre los que se puede pulsar y que conducen a obras que pueden consultarse libremente en otra página de internet": Sentencia del Tribunal de Justicia Europeo de 13 de febrero de 2014, Caso C-466/12, Nils Svenson y otros $v$. Retriever Sverige $A B$ (resaltado fuera de texto).

Es decir que si hubiese medidas de protección sobre la obra, esta no podría ser libremente consultada, pero si no las tiene, puede ser libremente accedida. Se puede inferir que la lógica de esta interpretación prejudicial es similar a la lógica del consentimiento implícito utilizado por la Suprema Corte Federal de Alemania en el sentido de que existe un consentimiento implícito si se coloca un contenido en la red sin medidas de protección con la idea de que no constituye un acto de comunicación pública si el enlace permite el acceso a un contenido que no tiene medidas de protección puesto que no se ha conformado un público nuevo.

36 Un análisis crítico de esta decisión prejudicial se encuentra en Mihaly Ficsor, "El derecho de comunicación al público (y puesta a disposición del público) a la luz de la jurisprudencia del Tribunal de Justicia de la Unión Europea”, Revista Iberoamericana 
En otros términos, la ausencia de restricción de acceso vendría a constituir una excepción al derecho exclusivo de puesta a disposición ${ }^{37}$.

De cualquier manera, no parece convincente el argumento de que por el hecho de que el autor coloque un contenido en la red sin medidas tecnológicas de protección, esté implícitamente autorizando su uso. No: a la luz del derecho continental ese uso debería obtener autorización previa de su titular. El hecho de colocar un contenido sin protección no significa que el derecho se haya agotado y que en consecuencia pueda ser utilizado libremente por otro actor o agente en la red, ya sea reproduciéndolo en forma reducida o comunicándolo al público ${ }^{38}$.

Por eso es por lo que esa interpretación puede ser entendida como una excepción al derecho de comunicación pública, en el sentido de que, puesto a disposición un contenido en la red, sin restricción, este podría ser comunicado por un motor de búsqueda, sin previa autorización.

De regreso al "consentimiento implícito" se puede decir que este argumento justificativo es discutible y que no encontraría apoyo dentro del sistema del derecho de autor de la Unión Europea ni dentro de sus países miembros. Tal como afirma Pötzlberger ${ }^{39}$, este argumento sería dentro del dilema de los thumbnail una respuesta válida solo de manera temporal. La excepción requeriría consagración normativa.

de Derecho de Autor, Cerlalc, Bogotá, año viI, n.o 14, enero-diciembre 2014, p. 66 a 97. Igualmente véase Herrera Sierra Luisa Fernanda, "El agotamiento del derecho de autor en la Jurisprudencia del Tribunal Europeo de Justicia”, disponible en: http:// propintel.uexternado.edu.co/derecho-autor/: "En el caso en concreto, el Tribunal concluyó que la 'publicación' mediante el hyperlinking no se encontraba orientada a un 'nuevo público' por cuanto los contenidos protegidos ya se encontraban en internet y por tanto, el concepto de 'público destinatario' integraba a todos los usuarios de la internet. Así pues, es posible vincular contenidos protegidos por el derecho de autor a través del uso de links cuando estos contenidos ya han sido comunicados al público y se encuentran disponibles en otros sitios web sin ninguna restricción".

37 Para Ficsor, "[1] introducción de una nueva excepción por el Tribunal sería obviamente inaceptable a la luz del hecho de que la Directiva de la Sociedad de la Información establece una lista exhaustiva de excepciones y limitaciones. El segundo problema sería aún más pesado, dado que establecer que el derecho de puesta a disposición del público se pierde en el entorno de Internet si los titulares de derecho no cumplen con una condición (restringir la disponibilidad de acceso), equivaldría a someter la aplicación de un derecho a una formalidad de facto": ob cit., p. 95 y 96.

38 "Under Svensson, once the work is accessible to 'all potential visitors to the site concerned,' i.e., for unrestricted sites, to all users of the internet, the author or rightholder may no longer invoke the communication to the public right to prohibit further making available of the work via the Internet, notably by linking or framing, even if these acts bring revenue to the linkers and framers. [...] an author who publishes, without restrictions, works on a website can neither have nor license exclusivity for this mode of exploitation as long as this initial act of exploitation lasts. As a result, the right of exploitation on the internet is severely truncated in a way unprecedented in copyright or any other intellectual property right, even as the internet is swiftly developing into the main exploitation market for works of authorship" (resaltado fuera de texto): ALAI 15 September 2013, Report on making available and communication to the public in the internet environment - focus on linking techniques on the internet, htt:// www.alai.org/en/assets/files/resolutions/making-available-rightreport-opinion.pdf; publicado asimismo en (2014) 36(3) EIPR 149.

39 Florian Pötzlberger, Google and the Thumbnail Dilemma - 'Fair use' in German Copyright Law? 9 ISJLP 139 2013-2014, p. 169. 
Frente a unas limitaciones y excepciones rígidas, se sostiene entonces que las excepciones flexibles son necesarias para capturar el actual proceso tecnológico que se vive. Sería pues indispensable incorporar elementos flexibles del fair use dentro del sistema europeo de las limitaciones y excepciones al derecho de autor.

En derecho europeo, en la Directiva 2001/29/CE, "relativa a la armonización de determinados aspectos de los derechos de autor y derechos afines a los derechos de autor en la sociedad de la información", existirían ciertos elementos conceptuales en sus considerandos que, con interpretaciones amplias, podrían justificar la actividad que desempeñan los motores de búsqueda ${ }^{40}$. Sin embargo, no existe una expresa o específica excepción aplicable a los motores de búsqueda, como se ha dicho.

\section{Derecho andino}

La Decisión Andina 351 de 1993 señala en el artículo 22 que "será lícito realizar, sin la autorización del autor y sin el pago de remuneración alguna, los siguientes actos: a) Citar en una obra, otras obras publicadas, siempre que se indique la fuente y el nombre del autor, a condición [de] que tales citas se hagan conforme a los usos honrados y en la medida justificada por el fin que se persiga”.

En el Proceso 139-IP-2003 se analizó si la reproducción de al menos tres cuartas partes de la obra del demandante, por parte del demandado, constituía un uso justo de la obra amparado por el derecho de cita. El Tribunal Andino de Justicia consideró al respecto: "Para la licitud de las citas es necesario que la obra de la que se toma el extracto haya sido lícitamente hecha, accesible al público, con anterioridad, que la cita se haga conforme a los hechos nombrados, que ella se haga en la medida justificada por el fin que se persiga y que se indique la fuente y el nombre del autor".

De modo pues que en nuestro contexto el derecho de cita aplicado con el estricto rigor que expone el Tribunal Andino no permitiría legitimar la actividad de reproducción o comunicación que realizan los motores de búsqueda de información.

40 Considerando 31: “Debe garantizarse un justo equilibrio entre los derechos e intereses de las diferentes categorías de titulares de derechos, así como entre las distintas categorías de titulares de derechos y usuarios de prestaciones protegidas. Las actuales excepciones y limitaciones a los derechos previstas en los Estados miembros deben revaluarse a la luz de los avances logrados en la electrónica". Considerando 35: "En determinados casos de excepciones o limitaciones, los titulares de los derechos deberían recibir una compensación equitativa para recompensarles adecuadamente por el uso que se haya hecho de sus obras o prestaciones protegidas". Considerando 44: "Al aplicar las excepciones y limitaciones previstas en la presente Directiva, estas deben ejercerse de acuerdo con las obligaciones internacionales. Las citadas excepciones y limitaciones no deben aplicarse ni de tal forma que los intereses legítimos del titular del derecho se vean perjudicados ni de manera contraria a la explotación normal de su obra o prestación. El establecimiento de dichas excepciones o limitaciones por los Estados miembros debe, en particular, reflejar debidamente el creciente impacto económico que puedan tener las mismas a la luz de los avances logrados en la electrónica" (resaltados fuera de texto). 


\section{LA REGLA DE LOS TRES PASOS}

Se ha argumentado que la estrictez de las limitaciones y excepciones podría flexibilizarse con la regla de los tres pasos en la medida en que en ella hay criterios abiertos que liberalmente interpretados permitirían ajustar el derecho de autor a la nueva realidad tecnológica ${ }^{41}$. En efecto, la regla de los tres pasos, de proveniencia anglosajona, introdujo criterios amplios cuya correcta aplicación podría adecuar la disciplina del derecho de autor al actual desarrollo tecnológico.

Obsérvese que la regla de los tres pasos utiliza criterios indeterminados que deben ser precisados por el juez, lo cual permitiría flexibilizar la aplicación del régimen de limitaciones y excepciones en los países de tradición de civil law o de derecho continental. Se ha observado que la regla de los tres pasos tiene sus raíces en la tradición del copyright anglosajón y que su interpretación amplia podría adecuar la regla a la actual realidad tecnológica. Por supuesto que la regla de los tres pasos ofrecería un "espacio de respiro" a las limitaciones cuando el titular del derecho de autor se halle adecuadamente compensado por la excepción del uso de un contenido no autorizado ${ }^{42}$.

Con los criterios abiertos que se utilizan en la regla de los tres pasos se podrían flexibilizar las limitaciones y excepciones al derecho de autor ${ }^{43}$. Sin embargo, el obstáculo persiste en la medida en que la regla de los tres pasos exige que la limitación solo opere en casos especiales, es decir, sin dar cabida a otro tipo de actos que pudieran ser tratados como excepción al derecho de autor pero que no han sido expresamente previstos por el legislador. El INDECOPI, por ejemplo, ha señalado:

... para que una conducta esté considerada dentro de los supuestos de limitación o excepción al derecho de explotación de los derechos de autor o derechos conexos no basta que esté expresamente contemplada como tal en la legislación de la materia, sino que además no debe atentar contra la explotación normal de la obra ni causar un perjuicio injustificado a los intereses del legítimo titular del derecho. Esto es lo que se conoce como la regla de los tres pasos o condiciones. Así, cualquier acto que no

41 En la Directiva 2001/29/CE la regla de los tres pasos se encuentra en el artículo 5.5: "Las excepciones y limitaciones contempladas en los apartados 1, 2, 3 y 4 se aplicarán en determinados casos concretos que no entren en conflicto con la explotación normal de la obra o prestación y no perjudiquen injustificadamente los intereses legítimos del titular del derecho". Casi con idéntico tenor la Decisión Andina en el artículo 21 señala: "Las limitaciones y excepciones al Derecho de Autor que se establezcan mediante las legislaciones internas de los Países Miembros, se circunscribirán a aquellos casos que no atenten contra la normal explotación de las obras o no causen perjuicio injustificado a los legítimos intereses del titular o titulares de los derechos" (resaltado fuera de texto).

42 "[T]he EC three-step test is moreover understood to offer enhanced breathing space for limitations when copyright owners are adequately compensated for exempted unauthorized use": Senftleben, ob. cit., p. 542.

43 "With the open-ended factors of special cases, normal exploitation, legitimate interests and unreasonable prejudice, the test itself is a source of inspiration for flexible law making in the field of copyright limitations rather than an obstacle to the introduction of national fair use systems": Senftleben, ob. cit., p. 548. 
cumpla con las condiciones antes mencionadas no podrá ser catalogado como un acto comprendido dentro de las limitaciones al derecho de autor, por lo que su realización deberá contar con la autorización previa del titular del derecho ${ }^{44}$.

Para otros, esto no sería obstáculo por cuanto la certeza legal no es necesariamente un trabajo exclusivo del legislador. Es decir que dicha labor puede ser compartida por legisladores y jueces. Lo cierto es que Senftleben, después de exponer en su estudio la rigidez del sistema de limitaciones, señala que es indispensable incorporar elementos del fair use en los sistemas nacionales de la Unión Europea:

Like in international copyright law, the three-step test would have to be perceived and used as a flexible balancing tool fulfilling different functions. [...] the test may serve as a means to reduce the scope of the limitation to reasonable proportions. [...] In this way, an appropriate copyright framework could be established, for instance, with regard, to advance search engine services, the digitization of cultural material and user-generated content ${ }^{45}$.

\section{Conclusiones}

En el fondo, la idea que subyace en el fair use es que en la mayoría de los casos una creación se basa en otra creación anterior; por ello, si con la obra derivada, así se tomen elementos de la anterior, hay un beneficio social, el juez con base en la doctrina del fair use legitima esa creación ${ }^{46}$.

Ahora bien, en la hora actual se perciben enfrentamientos entre nuevos modelos de negocios en internet con los titulares de derechos de autor y los proveedores de contenido. $\mathrm{Y}$ esos enfrentamientos y contradicciones deben ser resueltos por los jueces. Por eso estos tendrían la carga de contribuir con la certeza del sistema de las limitaciones.

En el sistema de las limitaciones y excepciones fijadas previamente por el legislador, y gobernado por la regla de los tres pasos, se observa una perspectiva de protección al autor por ser este la materia prima de la creación; en cambio, en el régimen del fair use, mucho más flexible, precisado por el juez, la idea que subyace es la del beneficio que la creación le preste a la sociedad ${ }^{47}$.

44 Instituto Nacional de Defensa de la Competencia y de la Protección de la Propiedad Intelectual (indecopi). Tribunal de Defensa de la Competencia y de la Propiedad Intelectual. Resolución No. 371-2001/TPI-INDECOPI, exp. 770-1999/odA.

45 Senftleben, ob. cit., p. 551.

46 La imitación, en ciertos casos, desde el punto de vista del mercado es pro-competitiva. Nótese que en derecho colombiano el artículo 14 de la Ley 256 de 1996, sobre competencia desleal, señala: "La imitación de prestaciones mercantiles e iniciativas empresariales ajenas es libre, salvo que estén amparadas por la ley".

47 En el caso de Google Books esto se aprecia en la medida que el alto beneficio social que implica la digitalización de millones de obras para facilitar su búsqueda justifica el fair use. La revista The Economist, comentando el fallo de apelación, señala: "How can a company get away with digitising millions of books without the authors' consent and showing them to the world? In his ruling, Judge Pierre Leval explained that copyright law gives 
Pero es conveniente indicar que incluso a la luz de sistemas jurídicos en donde la fuente principal es la ley tiene una gran injerencia el juez, por cuanto el sistema de las limitaciones y excepciones utiliza conceptos jurídicos indeterminados que deberán ser precisados por el mismo. ¿O es que acaso no lo son conceptos abiertos como el de "la normal explotación de las obras" o el del "perjuicio injustificado" al autor? Es el juez quien, con base en su fina intuición de lo justo, indicará cuándo el uso de una obra es honrado, esto es, que el uso no interfiere con la explotación normal de la obra ni causa un perjuicio injustificado a los intereses legítimos del autor.

No es pues válido en sentido absoluto afirmar que la diferencia entre los dos regímenes se encuentra en que el anglosajón es más flexible porque es un case by case approach, mientras que el otro es inflexible porque es diseñado por el legislador. En efecto, también en este la actividad del juez será fundamental en la determinación del alcance de las excepciones en el entorno digital.

Ahora bien, flexibilizar las limitaciones para recoger en ellas la actividad de los motores de búsqueda no implica desconocer los derechos que tiene el proveedor del contenido, y siendo así se haría necesario el reconocimiento de una remuneración o compensación equitativa para los titulares de derechos, o al menos el señalamiento normativo de los criterios para su fijación. Puesto que el derecho de autor es inherente al creador, ergo, el autor debe ser compensado por el uso no autorizado de su obra, y más, obviamente, si el motor de búsqueda obtiene ganancias con el uso no autorizado de contenidos.

¿Cuál sería el monto de esa remuneración?, y ¿quién la debe fijar, la autonomía privada de los implicados o el legislador? ${ }^{24}$. Este es el gran reto que tiene el derecho de autor con ocasión del uso de las obras protegidas en línea por los motores de búsqueda $^{49}$.

\footnotetext{
'potential creators' the exclusive right to copy their own work in order to expand everybody's 'access to knowledge'. It is not all about enriching authors. The 'ultimate, primary intended beneficiary', he wrote, 'is the public": The Economist, October 24th-30th 2015, p. 44.

48 El artículo 6 de la Directiva 2001/29 permite proteger el trabajo puesto a disposición del público online bajo términos contractuales y bajo las medidas tecnológicas de protección que previenen, entre otras cosas, la copia privada. Bajo tales condiciones, obsérvese, todas las limitaciones y excepciones podrían excluirse vía contractual: “Tanto las medidas tecnológicas adoptadas voluntariamente por los titulares de los derechos, incluidas las derivadas de acuerdos voluntarios, como las adoptadas en aplicación de medidas adoptadas por los Estados miembros, disfrutaran de la protección jurídica prevista en el apartado 1 ”.

49 El considerando 35 de la Directiva europea 2001/29 señala: "A la hora de determinar la forma, las modalidades y la posible cuantía de esa compensación equitativa, deben tenerse en cuenta las circunstancias de cada caso concreto. Un criterio útil para evaluar estas circunstancias sería el posible daño que el acto en cuestión haya causado a los titulares de los derechos. Cuando los titulares de los derechos ya hayan recibido una retribución de algún tipo, por ejemplo, como parte de un canon de licencia, puede ocurrir que no haya que efectuar un pago específico o por separado. El nivel de compensación equitativa deberá determinarse teniendo debidamente en cuenta el grado de utilización de las medidas tecnológicas de protección contempladas en la presente Directiva. Determinadas situaciones en las que el perjuicio causado al titular del derecho haya sido mínimo no pueden dar origen a una obligación de pago".
} 
En materia de motores de búsqueda fijar el monto de una licencia legal que recogiera el interés del tercero y el del titular de derecho sería una solución práctica dada la gran cantidad de imágenes que tienen indexadas los motores de búsqueda, lo cual hace imposible negociaciones individuales ${ }^{50}$. Pero este tema sería materia de otro escrito.

\section{BibLIOGRAFÍA}

ALAI, 15 de septiembre de 2013, Report on making available and communication to the public in the internet environment - focus on linking techniques on the internet, htt:// www.alai.org/en/assets/files/resolutions/making-available-rightreport-opinion.pdf; also published in (2014) 36(3) EIPR 149.

Associated Press v. Meltwater U.S. Holdings, Inc., 931 F. Supp. 2d 537, 540 (s.D.N.Y. 2013).

Authors Guild, Inc. v. Google Inc., 954 F. Supp 2d 282, 291 (s.D.N.Y. 2013).

Authors Guild, Inc. v. Google, Inc., No. 13-4829-cv (2d Cir. Oct. 16, 2015).

Bцом АмY, Search Engines and paragraph 512 (D) of the D.M.C.A, 1 Case W. Res. J.L. Tech. \& Internet 36 2009-2010.

Bohan, Faye, "Liability of Internet Search Engines", Hibernian Law Journal 6, n. ${ }^{\circ} 1$ (2006), 181-228.

Bunker, Matthew D. y Clay Calvert, "The Jurisprudence of transformation: Intellectual Incoherence and Doctrinal Murkiness Twenty Years after Campbell v. Acuff-Rose Music", Duke Law \& Technology Review.

Crews, Kenneth D., "The Role of the Judge in Common Law Countries When Deciding Fair Use Cases", Revista Iberoamericana de Derecho de Autor, Bogotá, CERLALC, año VIII, n. ${ }^{\circ} 15$, enero-junio 2015.

Ding Meng, "Perfect 10 v. Amazon.com: A Step Toward Copyright's Tort Law Roots”, Berkeley Technology Law Journal, vol. 23, n.o 1, February 2014.

Ficsor, Minaly, "El derecho de comunicación al público (y puesta a disposición del público) a la luz de la jurisprudencia del Tribunal de Justicia de la Unión Europea”, Revista Iberoamericana de Derecho de Autor, CERLALc, Bogotá, año VII, n. ${ }^{\circ}$ 14, enero-diciembre, 2014.

Grimmelman, James, "The Structure of Search Engine Law", The University of Iowa Law Review (The University of Iowa) 93 (2007).

Herrera Sierra, Luisa Fernanda. Estudio del Caso Google Books y efectos del fair use en el entorno digital. Disponible en: http://propintel.uexternado.edu.co/ estudio-del-caso-google-books-y-efectos-del-fair-use-en-el-entorno-digital/

Instituto Nacional de Defensa de la Competencia y de la Protección de

50 Las licencias legales tienen sentido cuando la negociación individual para una remuneración equilibrada no es práctica y tendría aplicación para muchos casos diferentes. Así mismo, como la recolección individual es difícil, esta labor la debe realizar una sociedad de gestión colectiva. 
la Propiedad Intelectual (Indecopi), Tribunal de Defensa de la Competencia y de la Propiedad Intelectual. Resolución n. o 371-2001/TPI-INDECOPI. EXPEDIENTE n. ${ }^{\circ}$ 770-1999/ODA.

Kelly v. Arriba Soft Corp., 336 F.3d 811, 817-22 (9 $9^{\text {th }}$ Cir. 2002).

New York Times Co. v. Tasini, 533 U.S. 483, 121 S. Ct. 2381, 150 L. Ed. $2 d 500$ (2001)

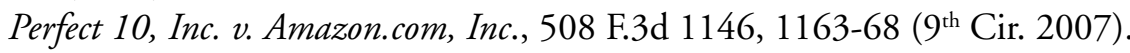

Pötzlberger, Florian, Google and the Thumbnail Dilemma- 'Fair use' in German Copyright Law? 9 isJLP 139, 2013-2014.

Ramírez, ÓsCaR, El equilibrio entre la propiedad intelectual y el acceso a la información y tecnología desde la perspectiva del usuario, Tesis de Maestría, Bogotá, Universidad Externado de Colombia, Departamento de Propiedad Intelectual, 2015.

Schonwald, Rosalina Jane, "Associated Press v. Meltwater US Holdings, Inc.; Fair Use, a Changing News Industry, and the Influence of Judicial Discretion and Custom", 29 Berkeley Tech. L.J. 799, 2014.

Senftleben, Martin, "Bridging the differences between copyright's legal traditions - the emerging EC fair use doctrine", 57 J. Copyright Soc'y USA 521, 2009-2010.

The Economist, "The 'ultimate, primary intended beneficiary", 13 de octubre de 2015.

Tribunal de Justicia Europeo, Sentencia del 13 de febrero de 2014, Caso C-466/12, Nils Svenson y otros v. Retriever Sverige AB. 